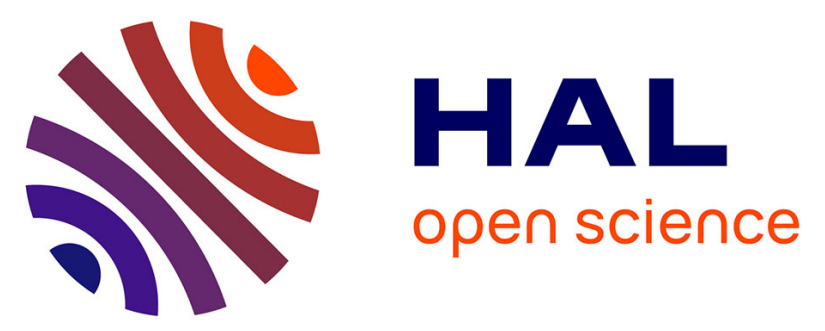

\title{
Optimal and low-complexity iterative joint network/channel decoding for the multiple-access relay channel
}

Xuan Thang Vu, Marco Di Renzo, Pierre Duhamel

\section{- To cite this version:}

Xuan Thang Vu, Marco Di Renzo, Pierre Duhamel. Optimal and low-complexity iterative joint network/channel decoding for the multiple-access relay channel. ICASSP 2011 - 36th IEEE International Conference on Acoustics, Speech and Signal Processing, May 2011, Prague, Czech Republic. pp.33123315, 10.1109/ICASSP.2011.5947093 . hal-00661315

\section{HAL Id: hal-00661315 \\ https://hal-centralesupelec.archives-ouvertes.fr/hal-00661315}

Submitted on 20 Jan 2012

HAL is a multi-disciplinary open access archive for the deposit and dissemination of scientific research documents, whether they are published or not. The documents may come from teaching and research institutions in France or abroad, or from public or private research centers.
L'archive ouverte pluridisciplinaire HAL, est destinée au dépôt et à la diffusion de documents scientifiques de niveau recherche, publiés ou non, émanant des établissements d'enseignement et de recherche français ou étrangers, des laboratoires publics ou privés. 


\title{
OPTIMAL AND LOW-COMPLEXITY ITERATIVE JOINT NETWORK/CHANNEL DECODING FOR THE MULTIPLE-ACCESS RELAY CHANNEL
}

\author{
Xuan-Thang Vu, Marco Di Renzo, and Pierre Duhamel \\ L2S, UMR 8506 CNRS - SUPELEC - Univ Paris-Sud \\ Laboratory of Signals and Systems (L2S), French National Center for Scientific Research (CNRS) \\ Ecole Supérieure d'Electricité (SUPELEC), University of Paris-Sud XI (UPS) \\ 3 rue Joliot-Curie, 91192 Gif-sur-Yvette (Paris), France \\ E-Mail: \{xuanthang.vu, marco.direnzo, pierre.duhamel\}@1ss.supelec.fr
}

\begin{abstract}
In this paper, we investigate joint network and channel decoding algorithms for the multiple-access relay channel. We consider a realistic reference scenario with Rayleigh fading over all the wireless links, including the source-torelay channels. Our contribution is twofold: i) first, we develop the quasi-optimal joint network and channel decoder by taking into account possible errors over the source-torelay channels, and ii) second, we propose a low-complexity iterative joint network and channel decoding algorithm, which reduces the number of channel decoders with respect to state-of-the-art solutions. Our numerical results show that: i) in fully-interleaved Rayleigh fading channels, the proposed solution provides almost the same bit error probability as the quasi-optimal scheme but with a reduction in complexity of approximately the $65 \%$, and ii) in Rayleigh block-fading channels, the proposed scheme yields almost the same bit error probability as state-of-the-art solutions but with a reduction in complexity of approximately the $30 \%$.
\end{abstract}

Index Terms - Network coding, joint network/channel decoding, iterative decoding.

\section{INTRODUCTION}

Network Coding (NC) is a recent field in electrical engineering and computer science, which allows the intermediate nodes of a network to combine several input packets into one or several output packets [1]. Recent results have shown that it can provide improved performance over conventional network routing techniques, as well as impact the design of the next-generation wireless ad hoc, sensor, and cellular networks, in terms of both energy efficiency and throughput. An overview of state-of-the-art and recent advances of NC for wireless networks is available in [2], [3]. However, besides the many potential advantages and applications of $\mathrm{NC}$ over classical routing, the $\mathrm{NC}$ principle is not without limitations. A fundamental problem that $\mathrm{NC}$ needs to take into account over lossy (e.g., wireless) networks is the so-called error propagation problem: corrupted packets injected by some intermediate nodes might propagate through the network until the destination, and might render impossible to decode the original information [4], [5].

Among the solutions that are currently being investigated to counteract the error propagation problem [2], Joint Network Channel Decoding (JNCD) is gaining a growing interest since its inception in [6], [7]. The basic premise of JNCD is the exploitation of the inherent redundancy of network and channel codes, in the same way as Joint Source and Channel Decoding (JSCD) exploits the inherent redundancy of source and channel codes [8]. In [6], [7], it has been shown that JNCD can improve the performance of canonical two-way and multiple-access relay channels if compared to conventional distributed turbo coding and separate network and channel decoding. Recent research works on JNCD have confirmed its potential gains with respect to layered schemes (see, e.g., [2], [9]-[11] and references therein).

In this paper, we study JNCD for the multiple-access Rayleigh fading channel by considering a realistic operating scenario with Additive White Gaussian Noise (AWGN) and channel fading over all the wireless links [12]. Our contribution is twofold. First, we develop the quasi-Optimal Maximum-Likelihood (ML) JNCD scheme (O-JNCD) for the multiple-access relay channel with XOR-based NC. Our implementation takes into account possible errors on the source-to-relay channels, which leads to a channel-aware implementation of the detector at the destination. It has been recently shown in [13] that a channel-aware receiver design can significantly improve the performance of NC. However, no channel coding is considered in [13]. We note that even though the O-JNCD might be too complicated for application in large networks, it is useful to set a lower-bound on the performance that can be achieved by sub-optimal JNCD schemes, as well as to understand the actual possibilities of improvement with respect to state-of-the-art solutions. Second, we propose a sub-optimal low-complexity iterative JNCD scheme, which is based on decoding principles of serial concatenated codes [14]. With respect to state-of-theart schemes for iterative JNCD [2], [6], [7], [9]-[11], our solution is based on propagating, between the blocks, extrinsic information of the coded bits, rather than extrinsic information of the information bits. This is achieved by providing a scheme that, as opposed to current solutions, starts the iterative decoding with the network code. We show that the scheme offers either the same or better performance than state-of-the-art approaches, but with a reduction in receiver complexity.

The reminder of this paper is organized as follows. In, Section 2, system model and notation are described. In Section 3, the channel-aware O-JNCD scheme is developed. In Section 4, the low-complexity iterative JNCD scheme is introduced and compared to the conventional scheme. In 
Section 5, some numerical results are shown to substantiate our claims. Finally, Section 6 concludes the paper.

\section{SYSTEM MODEL}

The system model under analysis is given by the canonical multiple-access relay channel, where two mobile stations, $\mathrm{MS}_{1}$ and $\mathrm{MS}_{2}$, communicate to a base station (BS) with the help of a relay $\mathrm{R}$ [7]. We study the realistic situation where all the channels are subject to Rayleigh fading and AWGN. In order to avoid mutual interference, we consider that transmissions are scheduled in time-orthogonal time-slots [2], [3]. The system is sketched in Fig. 1. The following notation is used: i) the information bits emitted by $\mathrm{MS}_{1}$ and $\mathrm{MS}_{2}$ at time-slot $k$ are denoted by $u_{1}(k)$ and $u_{2}(k)$, respectively; ii) the information bits are encoded by using a convolutional channel code, and $c_{j}(k)$ for $j=1,2$ denote the coded versions of $u_{j}(k)$, as follows:

$$
c_{j}(k)=\Gamma\left(u_{j}(k)\right) \text { and } u_{j}(k)=\Gamma^{-1}\left(c_{j}(k)\right), j=1,2
$$

where $\Gamma(\cdot)$ and $\Gamma^{-1}(\cdot)$ are short-hand for the encoding and decoding functions, respectively. Then, the coded bits are modulated and transmitted to R and BS. As a case study, Binary Phase Shift Keying (BPSK) modulation is considered. The Rayleigh-faded and noisy signals received at relay $\mathrm{R}$ are denoted by $x_{j R}(k)$ for $j=1,2$. The relay is assumed to perform channel decoding and ML-optimum demodulation by using the conventional BCJR algorithm [8], which is represented by the "SISO Dec" block in Fig. 1. The information bits estimated by the relay are denoted by $\hat{u}_{j R}(k)$ for $j=1,2$.

These estimated bits are network-coded by using, as a case study, XOR operations. The network coded information bit at time-slot $k$ is denoted by $u_{R}(k)=\hat{u}_{1 R}(k) \oplus \hat{u}_{2 R}(k)$, where $\oplus$ means bit-wise XOR. Then, these network-coded bits are channel-coded as follows:

$$
c_{R}(k)=\Gamma\left(u_{R}(k)\right)=\Gamma\left(\hat{u}_{1 R}(k) \oplus \hat{u}_{2 R}(k)\right)
$$

Finally, the network- and channel-coded bits in (2) are BPSK-modulated in a similar fashion as in $\mathrm{MS}_{1}$ and $\mathrm{MS}_{2}$. As a consequence, after three orthogonal time-slots, the destination receives three signals, as follows $(i=1,2, R)$ :

$$
x_{i}(k)=a_{i} c_{i}(k)+n_{i}(k)
$$

where i) $n_{i}(k) \sim N\left(0, \sigma_{i}^{2}\right)$ denotes the AWGN, which is assumed to be Guassian distributed with zero mean and variance $\sigma_{i}^{2}$, and ii) $a_{i}$ are the channel gains that are assumed to be independent and identically distributed with mean power $\mathrm{E}\left\{\left|a_{i}\right|^{2}\right\}=1$.

\section{O-JNCD SCHEME}

In this section, we aim at developing the O-JNCD decoder for the system model shown in Fig. 1. In particular, unlike common approaches (see, e.g., [12] for an overview), we take

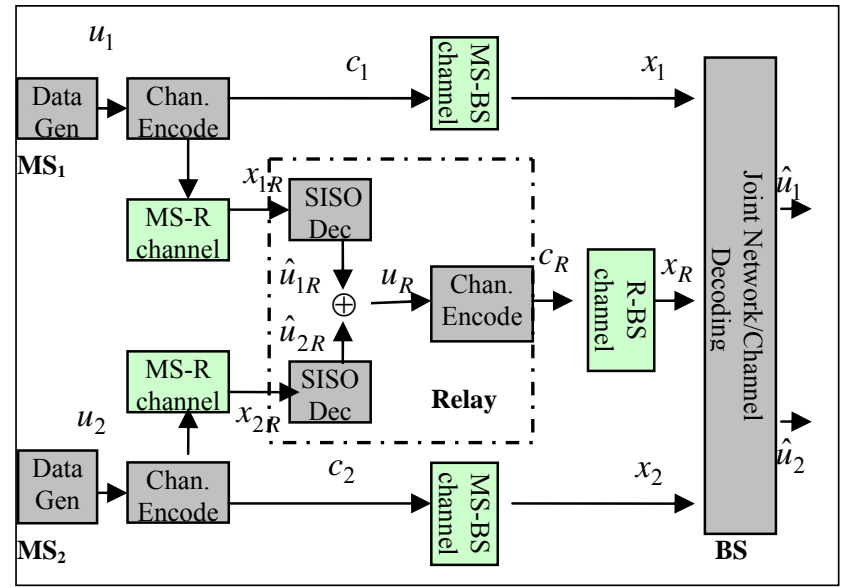

Fig. 1: Block diagram of system model.

into account possible errors on the source-to-relay links when developing our decoders. Furthermore, with respect to [13], channel coding is explicitly considered in our derivation.

More specifically, denoting by vectors $\mathbf{x}_{1}, \mathbf{x}_{2}, \mathbf{x}_{\mathrm{R}}$ a packet of received signals in (3), the $\mathrm{O}-\mathrm{JNCD}$ receiver according to the ML criterion can be mathematically stated as follows [8]:

$$
\begin{aligned}
{\left[\hat{u}_{1}(k), \hat{u}_{2}(k)\right] } & =\underset{\left\{u_{1}(k), u_{2}(k)\right\}}{\arg \max }\left\{\operatorname{Pr}\left\{u_{1}(k), u_{2}(k) \mid \mathbf{x}_{1}, \mathbf{x}_{2}, \mathbf{x}_{R}\right\}\right\} \\
& =\underset{\left\{u_{1}(k), u_{2}(k)\right\}}{\arg \max }\left\{\frac{\operatorname{Pr}\left\{u_{1}(k), u_{2}(k), \mathbf{x}_{1}, \mathbf{x}_{2}, \mathbf{x}_{R}\right\}}{\operatorname{Pr}\left\{\mathbf{x}_{1}, \mathbf{x}_{2}, \mathbf{x}_{R}\right\}}\right\}
\end{aligned}
$$

The analytical problem in (4) can efficiently be solved by using the Soft-Input-Soft-Output (SISO) module proposed in [15], which is useful for a generic iterative decoding problem of concatenated coding schemes (i.e., network and channel codes in our case). The key point for application in our case study is to consider that the trellis at the destination contains all the possible states of the two trellises in $\mathrm{MS}_{1}$ and $\mathrm{MS}_{2}$. Due to space constraints, we skip the details of the analytical derivation, and only mention that the forward filter, $\widetilde{\alpha}_{k}\left(S_{1}^{k}, S_{2}^{k}\right)$, and the backward filter, $\widetilde{\beta}_{k}\left(S_{1}^{k}, S_{2}^{k}\right)$, in the joint trellis (see [15] for details) can recursively be computed from the metric as follows:

$$
\begin{aligned}
\tilde{\gamma}_{k}\left(S_{1}^{k}, S_{2}^{k}, S_{1}^{k+1}, S_{2}^{k+1}\right)= & \operatorname{Pr}\left\{x_{1}(k) \mid c_{1}(k)\right\} \operatorname{Pr}\left\{x_{2}(k) \mid c_{2}(k)\right\} \\
& \times \operatorname{Pr}\left\{x_{R}(k) \mid c_{R}(k)\right\} \operatorname{Pr}\left\{u_{1}(k)\right\} \operatorname{Pr}\left\{u_{2}(k)\right\}
\end{aligned}
$$

where the probabilities $\operatorname{Pr}\left\{x_{1}(k) \mid c_{1}(k)\right\}, \operatorname{Pr}\left\{x_{2}(k) \mid c_{2}(k)\right\}$, $\operatorname{Pr}\left\{u_{1}(k)\right\}$, and $\operatorname{Pr}\left\{u_{2}(k)\right\}$ are known in closed-form for the system model in Section 2 and can be found in [8], [15]. On the other hand, the main difference, with respect to state-ofthe-art algorithms, is the term $\operatorname{Pr}\left\{x_{R}(k) \mid c_{R}(k)\right\}$, which accounts for the redundancy provided by the relay (spatial diversity) [3]. This term takes into account possible decoding errors at the relay node, which can be caused by Rayleigh fading and AWGN on the source-to-relay links [12]. Denoting by $P_{e}=\operatorname{Pr}\left\{c_{R} \neq\left(c_{1} \oplus c_{2}\right)\right\}$ the probability that the 
relay forwards erroneous bits, which is available in closedform in [16], the following result can be shown:

$$
\begin{aligned}
\sqrt{2} \sigma_{R} \operatorname{Pr}\left\{x_{R}(k) \mid c_{R}(k)\right\} & =\left(1-P_{e}\right) \exp \left(-\left|x_{R}(k)-c(k)\right|^{2} /\left(2 \sigma_{R}^{2}\right)\right)(6) \\
& +P_{e} \exp \left(-\left|x_{R}(k)+c(k)\right|^{2} /\left(2 \sigma_{R}^{2}\right)\right)
\end{aligned}
$$

The performance of this decoder is studied in Section 5.

\section{LOW-COMPLEXITY ITERATIVE JNCD SCHEME}

The aim of this section is to propose a new low-complexity iterative JNCD scheme for the scenario depicted in Fig. 1. First, let us start by summarizing the main working principle of the basic conventional JNCD (see, e.g., [2], [6], [7], [9][11]). A sketched block-diagram of the decoder is shown in Fig. 2. Basically, the solutions available in the literature foresee to perform iterative decoding by starting with channel decoding first, and then performing network decoding. In a few words, channel decoding is performed in a similar fashion as channel decoding at the relay node, by using the SISO module in Fig. 2. This block computes extrinsic information $L e_{u 1,2}^{D e c}$ and $L e_{u R}^{D e c}$ from the received signals $\mathbf{x}_{1}, \mathbf{x}_{2}$, $\mathbf{x}_{\mathrm{R}}$ and some a priori information [8], [14], [15]. Then, $L e_{u 1,2}^{D e c}$ and $L e_{u R}^{D e c}$ are input to the network decoder, which computes extrinsic information $L e_{u 1}^{N e t}$ and $L e_{u 2}^{N e t}$, which in turn are feedback to the channel decoders for the next iteration. The analytical details are here omitted, but have been developed by using the general treatment in [8], [14], [15].

Our proposed scheme, on the other hand, works in a different way. A simple block-diagram representation of our idea in shown in Fig. 3. The fundamental difference between Fig. 2 and Fig. 3 is that in the latter case network decoding is performed first. The fundamental advantage with respect to Fig. 2 is that one channel decoder can be avoided in our solution, which makes the receiver much simpler to be implemented in practice. Also, from the performance point of view, the two schemes are expected to be, in general, different, as the scheme in Fig. 2 propagates extrinsic information computed on the information bits, while the scheme in Fig. 3 propagates extrinsic information computed on the coded bits $L e_{c 1}^{N e t}$ and $L e_{c 2}^{N e t}$. This latter extrinsic information can be computed, at the $n$-th iteration, as follows:

$$
\left\{\begin{array}{l}
L e_{c 1}^{N e t(n)}=L_{x 1}+\log \left[\left(1+e^{L_{x R}+L_{x 2}+L L_{c 2}^{N e c t(n)}}\right) /\left(e^{L_{x R}}+e^{L_{x 2}+L a_{c 2}^{N e(n)}}\right)\right] \\
L e_{c 2}^{N e t(n)}=L_{x 2}+\log \left[\left(1+e^{L_{x R}+L_{x 1}+L a_{c 1}^{N e c(n)}}\right) /\left(e^{L_{x R}}+e^{L_{x 1}+L a_{c 1}^{N e(n)}}\right)\right]
\end{array}\right.
$$

where $L_{x 1}, L_{x 2}, L_{x R}$ are the log-likelihood of the a priori probabilities [8], [14], [15].

The fundamental analytical rationale for the scheme in Fig. 3 comes from the following chain of identities about the operations performed at the relay node $\mathrm{R}$ (i.e., network coding and channel coding):

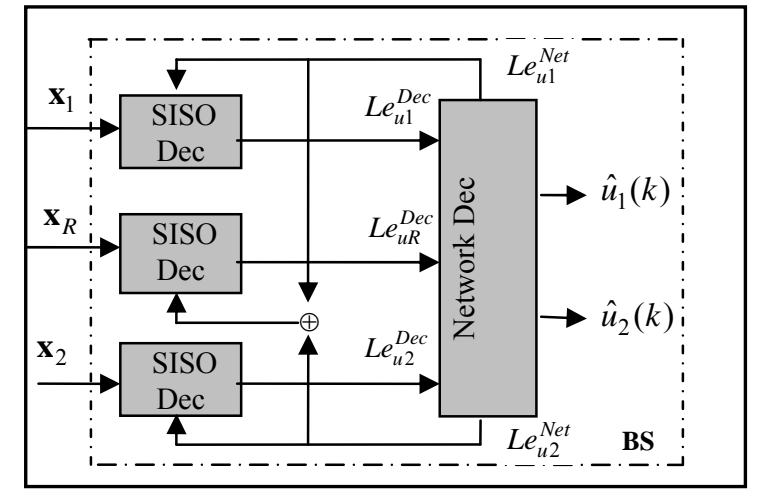

Fig. 2: Conventional JNCD scheme.

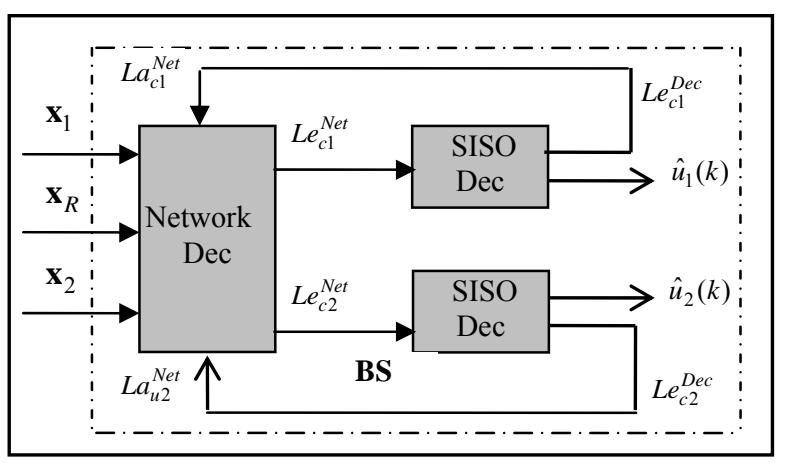

Fig. 3: Proposed low-complexity iterative JNCD scheme.

$$
\begin{aligned}
c_{R}(k) & =\Gamma\left(u_{R}(k)\right)=\Gamma\left(\hat{u}_{1 R}(k) \oplus \hat{u}_{2 R}(k)\right) \\
& =\Gamma\left(\hat{u}_{1 R}(k)\right) \oplus \Gamma\left(\hat{u}_{2 R}(k)\right)=\hat{c}_{1 R}(k) \oplus \hat{c}_{1 R}(k)
\end{aligned}
$$

which simply states that due to the linearity property of channel coding, NC can be performed directly on the estimated coded bits rather than on the estimated information bits. This provides the basic theoretical background for swapping the operations of channel and network decoding at the destination. While an accurate analytical investigation of the convergence issues and performance of the scheme in Fig. 3 is, due to space constraints, outside the scope of this paper, in Section 5 we provide some numerical results to substantiate the effectiveness of our solution. Furthermore, performance analysis is complemented with the study of the computational complexity.

\section{NUMERICAL RESULTS}

\subsection{Complexity}

Let $L$ be the constraint length of the channel code, $N$ be the block length, and $l$ be the number of iterations used. In Table 1 , we summarize the complexity in terms of multiplications and additions for the three schemes analyzed in this paper. We observe that the proposed scheme is the simplest to the implemented, and the complexity reduction with respect to the scheme in Fig. 2 comes from the fact that only two channel decoders are used at the destination. 
Table 1: Complexity. i) 2nd column: number of additions and multiplications; ii) 3rd column: ratio with the scheme in Fig. 2 when $L=3$ and $l=3$.

\begin{tabular}{|l|l|l|}
\hline JNCD (Fig. 2) & $9 l^{*} 2^{L+2}+14 l$ & 1 \\
\hline JNCD (Fig. 3) & $(3 l+1) * 2^{L+3}+16 l$ & 0.69 \\
\hline O-JNCD & $26 * 2^{2 L}+28$ & 1.97 \\
\hline
\end{tabular}

\subsection{Performance}

To compare the performance offered by the three schemes described above, we consider, as a case study, the following setup: i) a Recursive Systematic Convolutional (RSC) code with rate $R=1 / 2$ and polynomial generator $G=\left[\begin{array}{ll}13 & 15\end{array}\right]$, ii) a block length $N=600$ of information bits, which corresponds to a block of 1212 coded bits, iii) a symmetric multipleaccess relay channel where the relay is located at half distance between MSs and BS, which corresponds to a setup where the Signal-to-Noise Ratio (SNR) at the relay is $6 \mathrm{~dB}$ greater than the SNR at the destination, and iv) both blockfading and fully-interleaved fading channels are investigated. Selected numerical results for the Bit Error Rate (BER) are shown in Fig. 4. We notice that in fully-interleaved fading channels the proposed solution outperforms the conventional JNCD scheme in Fig. 2 (denoted as "Conventional" in the figure), and it is slightly worse than the O-JNCD scheme (which, although not being strictly optimal, is a lower-bound in our case). On the other hand, we observe that in blockfading channels our scheme provides the same BER as the scheme in Fig. 2, and they are, for large SNRs, both a few dB worse than the O-JNCD scheme. Thus, in this latter scenario possibilities of improvement are still possible and needed. In conclusion, by carefully analyzing the results summarized in Table 1 and Fig. 4, we note that the proposed JNCD scheme in Fig. 3 either provides the same BER as the conventional one but with a significant reduction in receiver complexity, or gives better performance by still keeping the same complexity reduction.

\section{CONCLUSION}

In this paper, we have studied joint network and channel decoding schemes for the multiple-access relay fading channels. Two solutions have been proposed and investigated. The O-JNCD provides a lower-bound when the destination has full channel knowledge of the overall network. The low-complexity JNCD scheme is, on the contrary, sub-optimal but provides close-to-optimal performance in fully-interleaved fading channels with a significant reduction in complexity. On the other hand, in block-fading channels it is a few $\mathrm{dB}$ worse than the optimal scheme, by providing the same BER as other iterative schemes available in the literature. However, its computational complexity is reduced. The comparison with the O-JNCD shows that possibilities of improvement are still possible in this scenario, especially for high SNRs.

\section{ACKNOWLEDGMENT}

The authors would like to thank Professor Nguyen Linh-Trung (Coltech, Hanoi) for helpful discussions.

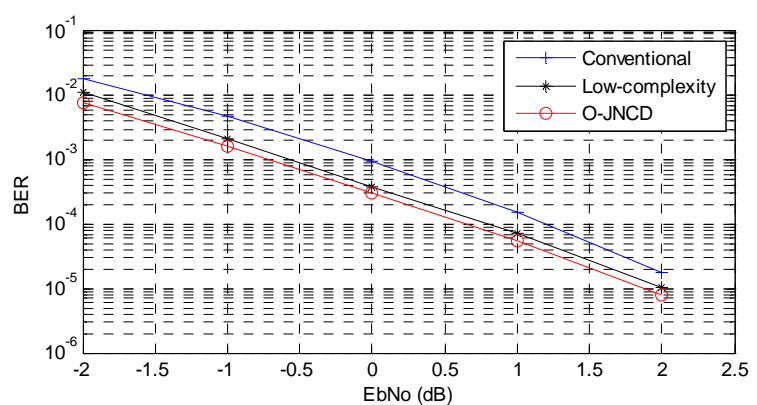

a) Fully-interleaved Rayleigh fading

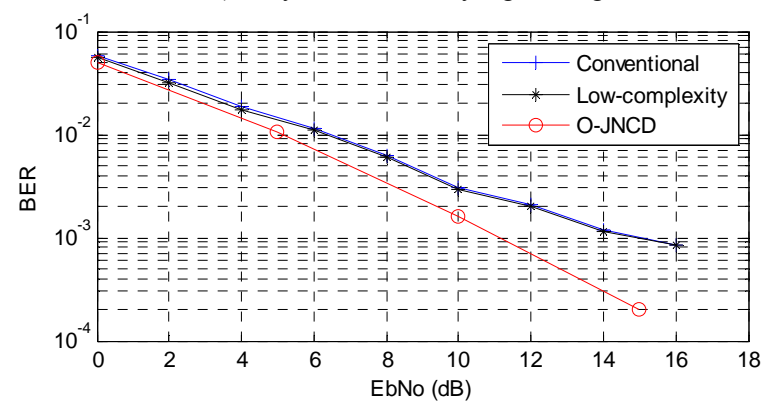

b) Block Rayleigh fading

Fig. 4: Performance comparison (BER).

\section{REFERENCES}

[1] R. Ahlswede et al., "Network information flow", IEEE Trans. Inform. Theory, vol.46, no.4, pp.1204-1216, July 2000.

[2] M. Di Renzo et al., "Robust wireless network coding - An overview", Springer Lecture Notes, pp. 685-698, 2010.

[3] M. Di Renzo, M. Iezzi, and F. Graziosi, "Beyond routing via network coding: An overview of fundamental information-theoretic results", IEEE PIMRC., Sept. 2010 (invited paper).

[4] P. A. Chou, Y. Wu, and K. Jain, "Practical network coding", Allerton Conf. Commun. Control, Computing, Oct. 2003.

[5] R. Koetter and F. R. Kschischang, "Coding for errors and erasures in random network coding”, IEEE Trans. Inform. Theory, vol. 54, no. 8, pp. 3579-3591, Aug. 2008

[6] C. Hausl and J. Hagenauer, "Iterative network and channel decoding for the two-way relay channel", IEEE. ICC, pp.1568-1573, June 2006.

[7] C. Hausl and P. Dupraz, "Joint network-channel coding for the multiple-access relay channel”, IEEE IWWN, pp. 817-822, Sept 2006.

[8] P. Duhamel and K. Kieffer, Joint source-channel decoding, Elsevier Dec. 2009, 334 pages.

[9] S. Zhang et al., "Joint design of network coding and channel decoding for wireless networks", IEEE WCNC, pp. 779-784, Mar. 2007.

[10] Z. Guo et al., "A practical joint network-channel coding scheme for reliable communication in wireless networks", ACM MobiHoc, pp 279-288, May 2009.

[11] Z. A. Plogar, M. P. Stef, and V. Bote, "Network and channel coded cooperation algorithms for cellular networks", IEEE VTC-Spring 2009.

[12] D. H. Woldegebreal and H. Karl, "Multiple-access relay channel with network coding and non-ideal source-relay channels", ISWCS, Oct. 2007.

[13] M. Xiao and T. Aulin, "Optimal decoding and performance analysis for a noisy channel network with network coding", IEEE Trans. Commun., vol. 57, no. 5, pp. 1402-1412, May 2009.

[14] P. A. Regalia, "Iterative decoding of concatenated codes: A tutorial", EURASIP J. Applied Signal Processing, pp. 762-774, 2005.

[15] S. Benedetto, D. Divsalar, G. Montorsi, and F. Pollara, "A soft-input soft-output APP module for iterative decoding of concatenated codes", IEEE Comm Lett., vol. 1, no. 1, pp. 22-24, Jan. 1997.

[16] M. Iezzi, M. Di Renzo, and F. Graziosi, "Network code design from unequal error protection coding: Channel-aware receiver design and diversity analysis", IEEE ICC, June 2011. 